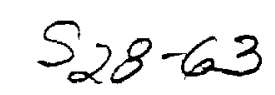

\title{
Vehicle Following Controller Design for Autonomous Intelligent Vehicles
}

N94- 30554

\author{
C. C. Chien, M. C. Lai and R. Mayr \\ Center for Advanced Transportation Technologies \\ Department of Electrical Engineering - Systems \\ University of Southern California \\ Los Angeles, CA 90089-2562
}

\begin{abstract}
A new vehicle following controller is proposed for autonomous intelligent vehicles. The proposed vehicle following controller not only provides smooth transient maneuver for unavoidable nonzero initial conditions but also guarantees the asymptotic platoon stability without the availability of feedforward information. Furthermore, the achieved asymptotic platoon stability is shown to be robust to sensor delays and an upper bound for the allowable sensor delays is also provided in this paper.
\end{abstract}

\section{INTRODUCTION}

Designing autonomous intelligent vehicles is important in the research of Advanced Vehicle Control Systems (AVCS) which is a major initiate in Intelligent Vehicle Highway Systems (IVHS). The main advantage of an autonomous intelligent vehicle is that it is considered as a "self-contained" system, i.e., it can operate together with other manually controlled vehicles without further technical assistance from highway infrastructure. Since future Automated Highway Systems (AHS) is planned to evolve from today's highway operation, the deployment of autonomous intelligent vehicles is of particular importance.

An autonomous intelligent vehicle is assumed to be capable of measuring (or estimating) necessary dynamical information from the immediate front vehicle by its on board sensors. The computer in the vehicle will then process these measured data and generate proper throttling and braking actions for controlling the vehicle's movement. These longitudinal maneuvers must be performed as swiftly as possible within the rider's comfort and safety constraints.

Traditionally, vehicle following controllers are designed for single-mass (triple integration) models which do not account for any propulsion system dynamics, see, e.g., $[1,6]$. In [8], Shladover included a simple first order engine model in the system dynamics and designed a linear vehicle following controller. It was shown that asymptotic platoon stability can be achieved by this linear controller when the drag forces (aerodynamic force and mechanical force) are neglected and the feedforward information is available. Based on the same vehicle model [8] with (nonlinear) drag forces taken into account, a nonlinear vehicle following controller was designed by Sheikholeslam and Desoer [7] using feedback linearization technique. In this case, asymptotic stability can also be achieved if the feedforward information is available. In [3], based on a more complicated vehicle engine model proposed in [5], Hedrick et al. proposed a sliding mode nonlinear controller to achieve vehicle following. The simulation results indicated that the controller has the potential of achieving asymptotic platoon stability if the feedforward information is available. This observation was later verified with proof in [9]. In [4], Ioannou and Chien modified the nonlinear vehicle following controller proposed in [7] and showed that asymptotic platoon stability can be achieved by this modified controller without any feedforward information. This result enhances the feasibility of the future deployment of autonomous intelligent vehicles.

In this paper, we propose a new vehicle following controller based on the nonlinear model proposed in [5] and [3]. The proposed vehicle following controller not only provides smooth transient maneuver for unavoidable nonzero initial conditions but also guarantees the asymptotic platoon stability without the availability of feedforward information. Furthermore, we show that the achieved asymptotic platoon stability is robust to sensor delay and an upper bound for the allowable sensor delays is provided.

This paper is organized as follows. In Section 2 and 3 , a vehicle longitudinal model and a safety distance policy are briefly reviewed. In Section 4, we present control methodologies for two classes of nonlinear control systems based on the ideas developed in backstepping control technique. Applying theses methodologies, we design vehicle 
following throttle and brake controller in Section 5. The issues of designing asymptotic platoon stability and its robustness to sensor delays are discussed in Section 6. In Section 7 , we use simulation results to demonstrate the effectiveness of our approach. At last, Section 8 gives a brief conclusion and possible future research directions.

\section{VEHICLE DYNAMICS MODEL}

In this section, we introduce a longitudinal powertrain model for control system design. The derivation of the system dynamic equations is based on the following assumptions [9]:

- Ideal gas law holds in the intake manifold.

- Temperature of the intake manifold does not change.

- There are no time delays in generating the power in the engine.

- The drive axle is sufficiently rigid.

- The torque converter is locked.

- The brakes follow first order dynamics.

The dynamics of the flow of air into and out of the intake manifold is described by

$$
\dot{m}_{a}=\dot{m}_{a i}-\dot{m}_{a o}
$$

where $m_{a}$ is the mass of air in the intake manifold and $\dot{m}_{a i}, \dot{m}_{a 0}$ are the mass flow rates through the throttle valve and into the cylinders, respectively.

Empirical equations developed for these flow rates are

$$
\begin{aligned}
\dot{m}_{a i} & =m_{a x} P_{R I}\left(m_{a}\right) T_{C}(\alpha) \\
\dot{m}_{a o} & =\dot{m}_{a o}\left(w_{e}, m_{a}\right)
\end{aligned}
$$

where $m_{a x}$ is a constant determined by the size of the intake manifold; $T_{C}(\cdot)$ is the throttle characteristic, a nonlinear function of the throttle angle $\alpha ; P_{R I}(\cdot)$ is the pressure influence function describing the choked flow relationship. Notice that $\dot{m}_{a 0}$ is generally measured by steadystate engine tests and supplied in tabular form as a function of the mass of air $m_{a}$ in the intake manifold and the engine speed $w_{e}$.

The engine's rotational dynamics is given by

$$
I \dot{w}_{e}=T_{n e t}\left(w_{e}, m_{a}\right)-R T_{b r}-R r F_{t r}
$$

where $I$ is the rotary inertia of the engine and the wheels referred to the engine side; $R$ is the effective gear ratio from the wheel to the engine; $T_{b r}$ is the brake torque; $T_{\text {net }}$ is the net-engine torque which is also measured by steady-state engine tests and supplied in tabular form as a function of $m_{a}$ and the engine speed $w_{e}$; $r$ is the effective tire radius; and $F_{t r}$ is the tractive force.
The tractive force can be expressed as

$$
F_{t r}=K_{r} \text { sat }(i / i)
$$

where $K_{r}$ is the longitudinal tire stiffness; $\bar{i}$ is a constant determined by the road and tire condition (usually around 0.15 [10]); sat $(\cdot)$ is the standard saturation function; and $i$ is the slip between the wheels and ground given by

$$
i=1-\frac{v}{R r w_{e}}
$$

In addition, we adopt a linear brake actuator model

$$
\dot{T}_{b r}=\frac{T_{b c}-T_{b r}}{\tau_{b}}
$$

where $\tau_{b}$ is the actuator time constant, $T_{b r}$ is the brake torque applied to the driven wheel and $T_{b c}$ is the commanded brake torque.

Finally, the longitudinal equation for the vehicle velocity is given by

$$
M \dot{v}=F_{t r}-c v^{2}-\mu M g
$$

where $c v^{2}$ is the aerodynamic drag, $\mu M g$ is the rolling resistance, and $M$ is the effective mass of the vehicle.

Under the "no-slip" condition [9], i.e.,

$$
v=R r w_{e},
$$

equations (1) and (2) yield

$$
J \dot{w}_{e}=T_{n e t}\left(w_{e}, m_{a}\right)-c R^{3} r^{3} w_{e}^{2}-R T_{b r}-\phi_{l}
$$

where $\phi_{l}=R r \mu M g ; J=I+M r^{2}$ is the effective inertia of the vehicle referred to the engine.

With above discussions, the $i^{t h}$ following vehicle has the following longitudinal dynamics,

$$
\begin{aligned}
\dot{x}_{i} & =v_{i}=R r w_{e} \\
\dot{w}_{e} & =\frac{1}{J}\left[T_{n e t}\left(w_{e}, m_{a}\right)-c R^{3} r^{3} w_{e}^{2}-R T_{b r}-\phi_{l}\right] \\
\dot{m}_{a} & =-\dot{m}_{a o}\left(w_{e}, m_{a}\right)+m_{a x} P_{R I}\left(m_{a}\right) T_{C}(\alpha) \\
\dot{T}_{b r} & =\frac{T_{b c}-T_{b r}}{\tau_{b}}
\end{aligned}
$$

where $x_{i}$ and $v_{i}$ denote the position and velocity along the longitudinal direction.

\section{Safety distance Policy}

For safe longitudinal operations, a following vehicle is required to keep a safe distance from its preceding vehicle. From the traffic capacity point of view, the desired safe distance should be as small as possible. However, the vehicle's performance capability, rider's comfort constraint and other safety considerations impose minimum bound on this distance. In this paper, we will adopt a desired safety distance policy [4] for the $i$ th following vehicle.

$$
S_{d_{i}}=\lambda_{1}\left(v_{i}^{2}-v_{i-1}^{2}\right)+\lambda v_{i}+\lambda_{3}
$$

where $\lambda_{1}, \lambda, \lambda_{3}$ are positive constants determined by the specified values of human reaction time, vehicle's full acceleration and deceleration, and maximal allowable jerk during deceleration.

While vehicle following is operating near a steady state the velocity of the control vehicle is approximately equal 
to the velocity of its preceding vehicle. Therefore, the safety distance policy can be well approximated by the constant time headway policy

$$
S_{d_{i}}=\lambda v_{i}+\lambda_{3}
$$

Let $x_{i}\left(x_{i-1}\right.$ resp.) and $v_{i}\left(v_{i-1}\right.$ resp.) be the position and velocity of the $i^{\text {th }}\left(i-1^{\text {th }}\right.$ resp.) vehicle. As shown in Figure (1), the spacing deviation for the $i$ th vehicle from the desired safety distance is

$$
\begin{aligned}
\delta_{i} & :=x_{i-1}-x_{i}-l_{i}-S_{d_{i}} \\
& =x_{i-1}-x_{i}-l_{i}-\lambda v_{i}-\lambda_{3}
\end{aligned}
$$

where $l_{i}$ is the length of controlled vehicle.

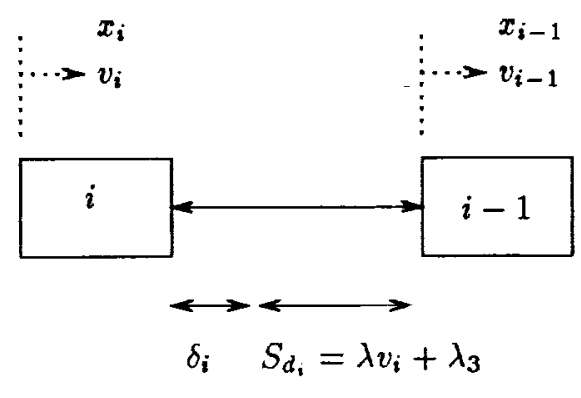

Figure 1:

For a group of vehicles with each vehicle's longitudinal dynamics described by (3) - (6), our control objective is to design a controller for each vehicle such that the following objectives are achieved: the spacing deviation $\delta_{i}$ can be regulated; the asymptotically platoon stability is achieved; and smooth transient response is guaranteed for non-zero initial spacing deviation and velocity deviation.

To this end, it seems that input-output feedback linearization technique may provide a promising approach to deal with this nonlinear control problem based on the structure of the system. However, since the mappings $T_{n e t}(\cdot, \cdot), m_{a o}(\cdot, \cdot)$ and $P_{R I}(\cdot)$ are supplied in tabular forms, their exact partial derivatives are not clearly identified. Consequently, feedback linearization method can not be applied directly.

\section{Nonlinear Control Methodologies}

In this section, we will show how the basic ideas used in backstepping control design approach can be applied to controller design for two classes of nonlinear systems. The control methodologies developed will then be used to design vehicle following controllers in Section V..

Nonlinear control systems Class I

Consider the following single-input single-output (SISO) nonlinear control system

$$
\begin{aligned}
\dot{x} & =f_{0}(w) \\
\dot{w} & =f_{1}(w, z) \\
\dot{z} & =f_{2}(w, z)+f_{3}(z) g_{1}(u) \\
y & =h\left(x, w, x_{m}, v_{m}\right)
\end{aligned}
$$

where $w, z \in \mathbf{R}$ are state variables; $y \in \mathbf{R}$ is the output; $f_{i}(i=0,1,2,3)$ and $g_{1}$ are smooth nonlinear functions; $\boldsymbol{x}_{m}, v_{m} \in \mathbf{R}$ are bounded external signals; and $u$ is the control input.

The control objective is to design the input $u$ so that the output $y$ is regulated, i.e., $\lim _{t \rightarrow \infty} y(t)=0$, while the state variables $w, z$ remain bounded. Our approach for finding an input $u$ to achieved the control objective is based on the application of control Lyapunov function in the backstepping technique developed in [2].

The basic ideas of applying backstepping technique to the control design for system (10) are roughly summarized in the following. First, we neglect the dynamics of state $z$ and treat $z$ as the input, then find a control input $z=z_{d}$ to achieve output regulation for the following reduced order system:

$$
\begin{aligned}
\dot{x} & =f_{0}(w) \\
\dot{w} & =f_{1}(w, z) \\
y & =h\left(x, w, x_{m}, v_{m}\right)
\end{aligned}
$$

Second, construct a state feedback $u$ from the computed $z_{d}$ such that

$$
\lim _{t \rightarrow \infty}\left(z(t)-z_{d}(t)\right)=0
$$

Finally, we show the control objective is achieved for the closed loop system.

For our approach, we make the following assumptions.

Assumption 4.1 $f_{3}(z)$ is nonzero and $\frac{\partial f_{1}}{\partial z}(w, z)$ is bounded for all $w, z \in \mathbf{R}$.

Assumption 4.2 The system

$$
\begin{aligned}
\dot{x} & =f_{1}(x, u) \\
y & =x
\end{aligned}
$$

is bounded input bounded output (BIBO) stable where $x, y, u \in \mathbf{R}$.

We now elaborate the control design procedure. Take

$$
V_{w}\left(x, w, x_{m}, v_{m}\right):=\frac{1}{2} \gamma_{1} h^{2}\left(x, w, x_{m}, v_{m}\right), \gamma_{1}>0
$$

as a Lyapunov function and evaluate the derivative of $V_{w}$ along the trajectory of (11). We get

$$
\begin{aligned}
& \dot{V}_{w}\left(x, w, x_{m}, v_{m}\right) \\
= & \gamma_{1} h \frac{d h}{d t} \\
= & \gamma_{1} h\left[\frac{\partial h}{\partial x} \dot{x}+\frac{\partial h}{\partial w} \dot{w}+\frac{\partial h}{\partial x_{m}} \dot{x}_{m}+\frac{\partial h}{\partial v_{m}} \dot{v}_{m}\right] \\
= & \gamma_{1} h\left[\frac{\partial h}{\partial x} f_{0}(w)+\frac{\partial h}{\partial w} f_{1}\left(w, z_{d}\right)+\frac{\partial h}{\partial x_{m}} \dot{x}_{m}+\frac{\partial h}{\partial v_{m}} \dot{v}_{m}\right]
\end{aligned}
$$


If, for $k_{1}>0, z_{d}$ is such that

$$
\begin{aligned}
f_{1}\left(w, z_{d}\right)= & \left(\frac{\partial h}{\partial w}\right)^{-1}\left[-k_{1} h-\frac{\partial h}{\partial x} f_{0}(w)\right. \\
& \left.-\frac{\partial h}{\partial x_{m}} \dot{x}_{m}-\frac{\partial h}{\partial v_{m}} \dot{v}_{m}\right]
\end{aligned}
$$

then

$$
\dot{V}_{w}\left(x, w, x_{m}, v_{m}\right)=-\gamma_{1} k_{1} h^{2}\left(x, w, x_{m}, v_{m}\right)
$$

For further developments, we will assume

Assumption 4.3 There exists a $z_{d}$ satisfying (12) for all $x, w, x_{m}, v_{m}$ in the domain of interest.

Take

$$
\begin{aligned}
& V_{\mathrm{u}}\left(x, w, z, z_{d}, x_{m}, v_{m}\right) \\
&:=\quad V_{w}\left(x, w, x_{m}, v_{m}\right)+\frac{1}{2} \gamma_{2}\left(z-z_{d}\right)^{2}, \quad \gamma_{2}>0 .
\end{aligned}
$$

as a Lyapunov function for (10). The derivative of $V_{u}$ along the trajectory of $(10)$ is

$$
\begin{array}{ll} 
& \dot{V}_{u}\left(x, w, z, z_{d}, x_{m}, v_{m}\right) \\
= & \gamma_{1} h\left[\frac{\partial h}{\partial x} f_{0}(w)+\frac{\partial h}{\partial w} f_{1}(w, z)+\frac{\partial h}{\partial x_{m}} \dot{z}_{m}\right. \\
& \left.+\frac{\partial h}{\partial v_{m}} \dot{v}_{m}\right]+\gamma_{2}\left(z-z_{d}\right)\left(\dot{z}-\dot{z}_{d}\right) \\
= & \gamma_{1} h\left[\frac{\partial h}{\partial x} f_{0}(w)+\frac{\partial h}{\partial w} f_{1}\left(w, z_{d}\right)+\frac{\partial h}{\partial x_{m}} \dot{x}_{m}\right. \\
& \left.+\frac{\partial h}{\partial v_{m}} \dot{v}_{m}\right]+\gamma_{1} h \frac{\partial h}{\partial w}\left[f_{1}(w, z)-f_{1}\left(w, z_{d}\right)\right] \\
& +\gamma_{2}\left(z-z_{d}\right)\left[f_{2}(w, z)+f_{3}(z) g_{1}(u)-\dot{z}_{d}\right] \\
=\quad & -\gamma_{1} k_{1} h^{2}+\gamma_{1} h \frac{\partial h}{\partial w}\left[f_{1}(w, z)-f_{1}\left(w, z_{d}\right)\right] \\
& +\gamma_{2}\left(z-z_{d}\right)\left[f_{2}(w, z)+f_{3}(z) g_{1}(u)-\dot{z}_{d}\right]
\end{array}
$$

If, for $k_{2}>0, u$ is such that

$$
\begin{aligned}
f_{3}(z) g_{1}(u)= & -k_{2}\left(z-z_{d}\right)-f_{2}(w, z)+\dot{z}_{d} \\
& -\frac{1}{\gamma_{2}\left(z-z_{d}\right)} \gamma_{1} h \frac{\partial h}{\partial w}\left[f_{1}(w, z)-f_{1}\left(w, z_{d}\right)\right]
\end{aligned}
$$

then

$$
\dot{V}_{u}\left(x, w, z, z_{d}, x_{m}, v_{m}\right)=-\gamma_{1} k_{1} h^{2}-\gamma_{2} k_{2}\left(z-z_{d}\right)^{2}
$$

Theorem 1 Consider the system (10) with the following proposed nonlinear state feedback controller

$$
\begin{aligned}
& u\left(x, w, z, x_{m}, v_{m}\right) \\
= & g_{1}^{-1}\left(\frac { 1 } { f _ { 1 } ( z ) } \left\{-k_{2}\left(z-z_{d}\right)-f_{2}(w, z)+\dot{z}_{d}\right.\right. \\
& \left.\left.-\frac{\gamma_{1}}{\gamma_{2}} \frac{1}{x-z_{d}} h \frac{\partial h}{\partial w}\left[f_{1}(w, z)-f_{1}\left(w, z_{d}\right)\right]\right\}\right)
\end{aligned}
$$

where $z_{d}$ satisfies (12). Suppose that Assumptions 4.1, 4.2 and 4.9 are satisfied. Then for the closed loop system (10), (12), we have $w$ remains bounded, $y$ converges to zero and $z$ converges to $z_{d}$ asymptotically.

Proof: Let

$$
\bar{z}:=z-z_{d}
$$

Then the closed loop syatem (10), (12) yields a subsystem

$$
\begin{aligned}
& \dot{h}=-k_{1} h+\frac{\partial h}{\partial w}\left[f_{1}\left(w, \bar{z}+z_{d}\right)-f_{1}\left(w, z_{d}\right)\right] \\
& \dot{\bar{z}}=-k_{2} \bar{z}-\frac{\gamma_{1}}{\gamma_{2}} \frac{1}{\bar{z}} h \frac{\partial h}{\partial w}\left[f_{1}\left(w, \bar{z}+z_{d}\right)-f_{1}\left(w, z_{d}\right)\right]
\end{aligned}
$$

By Assumption 4.1, we have

$$
\lim _{z \rightarrow z_{d}} \frac{f_{1}(w, z)-f_{1}\left(w, z_{d}\right)}{z-z_{d}}=\frac{\partial f_{1}}{\partial z}\left(w, z_{d}\right)<\infty
$$

This implies that $(h, \bar{z})=(0,0)$ is an equilibrium of the system (13), (14).

Take as a Lyapunov function for (13), (14).

$$
V(h, \bar{z}):=\frac{1}{2} \gamma_{1} h^{2}+\frac{1}{2} \gamma_{2} \bar{z}^{2},
$$

which is a positive definite, descrescent, and radially unbounded function. The derivative of $V$ along the trajectory of $(13),(14)$ is

$$
\begin{aligned}
\dot{V}= & \gamma_{1} h \dot{h}+\gamma_{2} \bar{z} \dot{\bar{z}} \\
= & -\gamma_{1} k_{1} h^{2}+\gamma_{1} h \frac{\partial h}{\partial w}\left[f_{1}(w, z)-f_{1}\left(w, z_{d}\right)\right]-\gamma_{2} k_{2} \bar{z}^{2} \\
& -\gamma_{1} h \frac{\partial h}{\partial w}\left[f_{1}(w, z)-f_{1}\left(w, z_{d}\right)\right] \\
= & -\gamma_{1} k_{1} h^{2}-\gamma_{2} k_{2} \bar{z}^{2} \\
< & 0
\end{aligned}
$$

Therefore, we see

$$
h, \bar{z} \in L_{2} \cap L_{\infty}
$$

The boundedness of $w$ can be established by the boundedness of $\bar{z}$ and Assumptions 4.2 and 4.3. Finally, from the well known lyapunov theorem, we conclude that $h$ converges to zero and $z$ converges to $z_{d}$ asymptotically.

Nonlinear control systems: Class II We now consider the nonlinear control system

$$
\begin{aligned}
\dot{x} & =f_{0}(w) \\
\dot{w} & =f_{1}(w, z)+f_{1}(\eta) \\
\dot{z} & =f_{2}(w, z) \\
\dot{\eta} & =f_{3}(\eta)+g_{1}(u) \\
y & =h\left(x, w, x_{m}, v_{m}\right)
\end{aligned}
$$

where $x, w, z \eta \in \mathbf{R}$ are state variables; $y \in \mathbf{R}$ is the output; $f_{i}(i=0,1,2,3,4)$ and $g_{1}$ are smooth nonlinear functions; $x_{m}, v_{m} \in \mathbf{R}$ are bounded external signals; and $u$ is the control input.

The control objective is to design input $u$ so that the output $y$ is regulated while the state variables $w, z, \eta$ remain bounded. We assume

Assumption $4.4 \frac{\partial f_{4}}{\partial \eta}(\eta)$ is bounded.

Assumption 4.5 The system

$$
\begin{aligned}
& \dot{x}_{1}=f_{1}\left(x_{1}, x_{2}\right)+f_{4}(u) \\
& \dot{x}_{2}=f_{2}\left(x_{1}, x_{2}\right) \\
& y=\left[\begin{array}{ll}
x_{1} & x_{2}
\end{array}\right]^{T}
\end{aligned}
$$

is $\mathrm{BIBO}$ stable. 
The control design for (15) is similar to the one for system (10). To start with, we neglect the dynamics of state $\eta$ and treat $\eta$ as the control input of the system (15), then try to find a control $\eta_{d}$ to achieve control objective for the following reduced order system:

$$
\begin{aligned}
\dot{x} & =f_{0}(w) \\
\dot{w} & =f_{1}(w, z)+f_{4}\left(\eta_{d}\right) \\
\dot{z} & =f_{2}(w, z) \\
y & =h\left(x, w, x_{m}, v_{m}\right)
\end{aligned}
$$

Take

$$
V_{w}\left(x, w, x_{m}, v_{m}\right)=\frac{1}{2} \gamma_{3} h^{2}\left(x, w, x_{m}, v_{m}\right), \gamma_{3}>0
$$

as a Lyapunov function and evaluate the derivative of $V_{w}$ along the trajectory of $(19)$. We get

$$
\begin{aligned}
& \dot{V}_{w}\left(x, w, x_{m}, v_{m}\right) \\
= & \gamma_{3} h\left[\frac{\partial h}{\partial x} \dot{x}+\frac{\partial h}{\partial w} \dot{w}+\frac{\partial h}{\partial x_{m}} \dot{x}_{m}+\frac{\partial h}{\partial v_{m}} \dot{v}_{m}\right] \\
= & \gamma_{3} h\left\{\frac{\partial h}{\partial x} f_{0}(w)+\frac{\partial h}{\partial w}\left[f_{1}(w, z)+f_{1}\left(\eta_{d}\right)\right]\right. \\
& \left.+\frac{\partial h}{\partial x_{m}} \dot{x}_{m}+\frac{\partial h}{\partial v_{m}} \dot{v}_{m}\right\}
\end{aligned}
$$

If, for $k_{3}>0, \eta_{d}$ is such that

$$
\begin{aligned}
f_{4}\left(\eta_{d}\right)= & -f_{1}(w, z)+\left(\frac{\partial h}{\partial w}\right)^{-1}\left[-k_{3} h-\frac{\partial h}{\partial x} f_{0}(w)\right. \\
& \left.-\frac{\partial h}{\partial x_{m}} \dot{x}_{m}-\frac{\partial h}{\partial v_{m}} \dot{v}_{m}\right]
\end{aligned}
$$

then

$$
\dot{V}_{w}\left(x, w, x_{m}, v_{m}\right)=-\gamma_{3} k_{3} h^{2}
$$

Similarly, we assume

Assumption 4.6 There exists an $\eta_{d}$ satisfying (20) for all $x, w, z, x_{m}, v_{m}$ in the domain of interest.

With Assumption 4.6, we take

$$
V_{u}\left(x, w, \eta, \eta_{d}, x_{m}, v_{m}\right)=V_{w}\left(x, w, x_{m}, v_{m}\right)+\frac{1}{2} \gamma_{4}\left(\eta-\eta_{d}\right)^{2}
$$

as a Lyapunov function and evaluate its derivative along the system (15). We have

$$
\begin{array}{ll} 
& \dot{V}_{u}\left(x, w, \eta_{1} \eta_{d}, x_{m}, v_{m}\right) \\
= & \gamma_{3} h\left\{\frac{\partial h}{\partial x} f_{0}(w)+\frac{\partial h}{\partial w}\left[f_{1}(w, z)+f_{4}\left(\eta_{d}\right)+f_{4}(\eta)-f_{4}\left(\eta_{d}\right)\right]\right. \\
& \left.+\frac{\partial h}{\partial x_{m}} \dot{x}_{m}+\frac{\partial h}{\partial v_{m}} \dot{v}_{m}\right\}+\gamma 4\left(\eta-\eta_{d}\right)\left(\dot{\eta}-\dot{\eta}_{d}\right) \\
= & -\gamma_{3} k_{3} h^{2}+\gamma_{3} h \frac{\partial h}{\partial w}\left[f_{4}(\eta)-f_{4}\left(\eta_{d}\right)\right] \\
& +\gamma 4\left(\eta-\eta_{d}\right)\left[f_{3}(\eta)+g_{1}(u)-\dot{\eta}_{d}\right]
\end{array}
$$

If, for $k_{4}>0, u$ is such that

$$
\begin{aligned}
g_{1}(u)= & -k_{4}\left(\eta-\eta_{d}\right)-f_{3}(\eta)+\dot{\eta}_{d} \\
& -\frac{1}{\gamma_{4}\left(\eta-\eta_{d}\right)} \gamma_{3} h \frac{\partial h}{\partial w}\left[f_{4}(\eta)-f_{4}\left(\eta_{d}\right)\right]
\end{aligned}
$$

then

$$
\dot{V}_{u}\left(x, w, \eta, \eta_{d}, x_{m}, v_{m}\right)=-\gamma_{3} k_{3} h^{2}-\gamma_{4} k_{4}\left(\eta-\eta_{d}\right)^{2}
$$

Theorem 2 Consider the system (15) with the following proposed nonlinear state feedback controller

$$
\begin{aligned}
& u\left(x, w, z, \eta, x_{m}, v_{m}\right) \\
= & g_{1}^{-1}\left(-k_{4}\left(\eta-\eta_{d}\right)-f_{3}(\eta)+\dot{\eta}_{d}\right. \\
& \left.-\frac{1}{\gamma_{4}\left(\eta-\eta_{d}\right)} \gamma_{3} h \frac{\partial h}{\partial w}\left[f_{4}(\eta)-f_{4}\left(\eta_{d}\right)\right]\right)
\end{aligned}
$$

where $\eta_{d}$ satisfies (20). Suppose that Assumptions 4.4, 4.5 and 4.6 are satisfied. Then for the closed loop system (15), (21), we have $w, z$ remain bounded, $y$ converges to zero and $\eta$ converges to $\eta_{d}$ asymptotically.

Proof: The proof is similar to theorem 1.

\section{Vehicle Following Controller Design}

A vehicle following controller is required to maintain a desired spacing between vehicles and to guarantee asymptotic platoon stability. The property that the spacing error for a controlled vehicle can be regulated is referred to local stability. A platoon is asymptotically stable if there are no slinky-type effects [7] within a platoon. Researchers have found that local stability in vehicle following is not enough to guarantee asymptotic platoon stability. Moreover, the unavoidable non-zero initial conditions occurring during various mode transitions, e.g., switching from manually control to automatic control, can generate transient torque large enough to degrade the driving quality.

In this section, the control methodologies developed in Section IV are applied to design a vehicle following controller with local stability and asymptotic platoon stability. To deal with the undesirable transient response caused by non-zero initial conditions, we will filter the desired control effort by introducing an imaginary preceding vehicle in the controller design. Stability is guaranteed by the fact that the states of the imaginary preceding vehicle will converge to that of the true preceding vehicle exponentially and the (imaginary) spacing deviation (from the desired spacing between the imaginary vehicle and the controlled vehicle) is regulated. With properly chosen design parameters, the proposed controller achieves asymptotic platoon stability which is robust to sensor delays.

\section{A. Controller Design}

The proposed controller is composed of a throttle controller, a brake controller, and a switching logic. The brake controller is to execute the decelerating operation. The throttle controller is to perform the accelerating and decelerating maneuvers while braking is not required for assistance. The switching logic is to properly activate and deactivate the throttle and brake controllers based on the needed control action at the current operating state. To be precise, the controller will continuously compute the required throttle angle required by the control action. If the 
calculated required throttle angle is greater than the minimum throttle angle, say $\alpha_{0}$, the logic determines that the throttle controller alone is capable of handling the desired maneuver, and no brake torque is to be applied. If not, the logic will deactivate the throttle controller, i.e., keep the throttle angle at $\alpha_{0}$, and activate the brake controller to generate the proper brake torque.

To smooth the transient response during vehicle maneuvering, we introduce for the $i^{\text {th }}$ (following) vehicle an imaginary preceding vehicle with dynamics characterized by the following equations

$$
\begin{aligned}
\dot{\bar{x}}_{i-1} & =\hat{v}_{i-1} \\
\dot{\hat{v}}_{i-1} & =-\beta_{2}\left(\hat{v}_{i-1}-v_{i-1}\right)-\beta_{1}\left(\hat{x}_{i-1}-x_{i-1}\right) \\
\hat{x}_{i-1}(0) & =x_{i}(0)+l_{i}+\lambda v_{i}(0)+\lambda_{3} \\
\hat{v}_{i-1}(0) & =v_{i}(0)
\end{aligned}
$$

where $\hat{x}_{i-1}, \hat{v}_{i-1}$ can be viewed as the position and velocity of the imaginary proceding vehicle for the $i^{\text {th }}$ vehicle; $\beta_{1}=\beta_{1}\left(\delta_{i}(0), v_{i-1}(0)-v_{i}(0)\right)$ and $\beta_{2}=\beta_{2}\left(\delta_{i}(0), v_{i-1}(0)-\right.$ $\left.v_{i}(0)\right)$ are positive functions of $\delta_{i}(0)$ and $\left(v_{i-1}(0)-v_{i}(0)\right)$ to be specified by designers.

Remark 5.1 It is easily verified that if $\dot{v}_{i-1}=0$, i.e., the (true) preceding vehicle is traveling at constant velocity, it can be easily shown that $\left(\hat{x}_{i-1}-x_{i-1}\right)(t)$ and $\left(\hat{v}_{i-1}-v_{i-1}\right)(t)$ converge to 0 exponentially. With suitably chosen parameters $\beta_{1}$ and $\beta_{2}$, we can have proper convergence property of $\left(\hat{x}_{i-1}-x_{i-1}\right)(t)$ and $\left(\hat{v}_{i-1}-v_{i-1}\right)(t)$.

Remark 5.2 Negative $\delta_{i}(0)$ or $v_{i-1}(0)-v_{i}(0)$ may lead to the situation that the imaginary preceding vehicle is traveling ahead of the true preceding vehicle. For large negative value of $\delta_{i}(0)$ or $v_{i-1}(0)-v_{i}(0)$, which is possibly an indication of impending collision, it is necessary to reflect this situation to the controller as soon as possible (which enables the controller of the controlled vehicle to be able to respond it properly for avoiding collision). Therefore, the values of $\beta_{1}$ and $\beta_{2}$ should be chosen in the sense that fast convergence rate is assured.

Define

$$
\hat{\delta}_{i}:=\hat{x}_{i-1}-x_{i}-\lambda v_{i}-l_{i}-\lambda_{3} .
$$

Compared (23) with (9), $\hat{\delta}_{i}$ can be regarded as the deviation of the desired spacing between the imaginary vehicle and the controlled vehicle. Furthermore, we see from (22)

$$
\hat{\delta}_{i}(0)=0 \text {. }
$$

In order to shape the desired transient response, we adopt the idea of PID control and define a function to be regulated

$$
h:=c_{p} \hat{\delta}_{i}+c_{I} \int_{0}^{t} \hat{\delta}_{i} d \xi+\left(\hat{v}_{i-1}-v_{i}\right)
$$

where $c_{p}$ and $c_{I}$ are design parameters to be determined.

The design of throttle and brake controllers are discussed separately in the following.
Vehicle following throttle controller

Under the condition that the brake controller is deactivated, the vehicle longitudinal dynamic equations are reduced to

$$
\begin{aligned}
\dot{x}_{i} & =v_{i}=R r w_{e} \\
\dot{w}_{e} & =\frac{1}{J}\left[T_{\text {net }}\left(w_{e}, m_{a}\right)-c R^{3} r^{3} w_{e}^{2}-\phi_{l}\right] \\
\dot{m}_{a} & =-\dot{m}_{a o}\left(w_{e}, m_{a}\right)+m_{a x} P_{R I}\left(m_{a}\right) T_{C}(\alpha)
\end{aligned}
$$

We see that the system (25) - (27) with output function $h$ given in (24) can be represented by equation (10) with the following variable and function substitutions

$$
\begin{aligned}
& (x, w, z, u)=\left(x_{i}, w_{e}, m_{a}, \alpha\right), \\
& f_{0}(w)=R r w, \\
& f_{1}(w, z)=\frac{1}{J}\left[T_{n e t}(w, z)-c R^{3} r^{3} w^{2}-\phi_{l}\right], \\
& f_{2}(w, z)=-\dot{m}_{a o}(w, z), \\
& f_{3}(z)=m_{a x} P_{R I}(z), \quad\left(x_{m}, v_{m}\right)=\left(\hat{x}_{i-1},\right. \\
& \left.\hat{v}_{i-1}\right), \quad g_{1}(u)=T_{c}(u) \\
& h=\left(v_{m}-R r w\right)+c_{p}\left(x_{m}-x-\lambda R r w\right) \\
& +c_{I} \int_{0}^{t}\left(x_{m}-x-\lambda R r w\right)(\xi) d \xi
\end{aligned}
$$

It is further verified that Assumptions 4.1, 4.2 are satisfied. Besides, the Assumptions 4.3 is also satisfied in the range of operation. By Theorem 1, we propose the following control law

$$
=T_{c}^{-1}\left(\begin{array}{c}
\frac{1}{m_{a x} P_{R I}\left(m_{e}\right)}\left\{-k_{2}\left(m_{a}-m_{a, d e s}\right)+\dot{m}_{a o}\left(w_{e}, m_{a}\right)\right. \\
+\dot{m}_{a, \text { des }}+\frac{\gamma_{2}}{\gamma_{2}} \frac{\left(1+\lambda c_{p}\right) R r}{J} \\
\frac{h}{m_{a}-m_{a, d e s}}\left[T_{n e t}\left(w_{e}, m_{a}\right)-T_{n e t}\left(w_{e}, m_{a, d e s}\right]\right\}
\end{array}\right)
$$

where $m_{a, d e s}$ satisfies

$$
\begin{aligned}
& T_{n e t}\left(w_{e}, m_{a, d e s}\right) \\
= & \frac{J}{\left(1+\lambda c_{p}\right) R r}\left[c_{p}\left(\hat{v}_{i-1}-v_{i}\right)+\left(c_{I}+k_{1} c_{p}\right) \hat{\delta}_{i}\right. \\
& +k_{1} c_{I} \int_{0}^{t} \hat{\delta}_{i} d \xi-\beta_{2}\left(\hat{v}_{i-1}-v_{i}\right)-\beta_{1}\left(\hat{x}_{i-1}-x_{i}\right) \\
& \left.+k_{1}\left(\hat{v}_{i-1}-v_{i}\right)\right]+\phi_{l}
\end{aligned}
$$

From Theorem 1, it is clear to see

Proposition 3 Consider the system (25)-(27). The controller proposed in (28) - (28) will drive $h$ to zero asymptotically.

While implementing the control law (28), $\dot{m}_{a, d e s}$ is to be estimated by finite differencing sampling values of $m_{a, d e s}$.

We will delay the discussion of the convergence of $\delta_{i}(t)$ until the brake controller is presented since in both control schemes we can show the same convergence property of $\delta_{i}(t)$

Vehicle following brake controller

When the brake controller is activated, the throttle angle is kept at the minimum $\alpha_{0}$. In this case, the vehicle's dynamics is governed by equations (3) - (6) with $\alpha$ replaced 
by the constant $\alpha_{0}$. Notice that the system (3) - (6) with output function (24) can be represented by (15) with the following variable and function substitutions

$$
\begin{aligned}
& (x, w, z, \eta, u)=\left(x_{i}, w_{e}, m_{a}, T_{b r}, T_{b c}\right), \quad f_{0}(w)=R r w, \\
& f_{1}(w, z)=\frac{1}{J}\left[T_{n e t}(w, z)-c R^{3} r^{3} w^{2}-\phi_{l}\right], \quad f_{4}(\eta)=-\frac{R}{J} \eta \\
& f_{2}(w, z)=-\dot{m}_{a o}(w, z), \quad\left(x_{m}, v_{m}\right)=\left(\hat{x}_{i-1}, \hat{v}_{i-1}\right), \\
& f_{3}(\eta)=-\frac{1}{\tau_{b}} \eta, \quad g_{1}(u)=\frac{1}{\tau_{b}} u, \\
& h=\left(v_{m}-R r w\right)+c_{p}\left(x_{m}-x-\lambda R r w\right) \\
& +c_{I} \int_{0}^{t}\left(x_{m}-x-\lambda R r w\right)(\xi) d \xi
\end{aligned}
$$

In addition, Assumptions 4.4, 4.5 are satisfied. And the Assumptions 4.6 is also satisfied in the operating range.

To regulate the output function (24), we propose the following brake control law

$$
\begin{aligned}
T_{b c}= & \tau_{b}\left[-k_{4}\left(T_{b r}-T_{b r, d e s}\right)+\frac{1}{\tau_{b}} T_{b r}+T_{b r, \text { des }}\right. \\
& \left.-\frac{\gamma_{3}}{\gamma_{4}} \frac{\left(1+\lambda c_{p}\right) R^{2} r}{J} h\right]
\end{aligned}
$$

where

$$
\begin{aligned}
T_{b r, d e s} & = \\
& \frac{1}{R}\left[T_{\text {net }}\left(w_{e}, m_{a}\right)-\phi_{l}\right]-\frac{J}{R^{2} r\left(1+\lambda c_{p}\right)}\left[c_{p}\left(\hat{v}_{i-1}-v_{i}\right)\right. \\
& +\left(c_{I}+k_{1} c_{p}\right) \hat{\delta}_{i}+k_{1} c_{I} \int_{0}^{t} \hat{\delta}_{i} d \xi-\beta_{1}\left(\hat{v}_{i-1}-v_{i}\right) \\
& \left.-\beta_{2}\left(\hat{x}_{i-1}-x_{i}\right)+k_{1}\left(\hat{v}_{i-1}-v_{i}\right)\right]+\phi_{l}
\end{aligned}
$$

By Theorem 3, we see

Proposition 4 Consider the system (3)-(6) with $\alpha=\alpha_{0}$ and output function (24). The controller proposed in (29)(30) will drive $h$ to zero asymptotically.

Similarly, $\dot{T}_{b r, d e s}$ is to be computed numerically by finite difference sampling values of $T_{b r, d e s}$.

\section{Regulation of $\delta_{i}$}

Recall that our goal is to regulate the spacing deviation $\delta_{i}$ in both throttle and brake control cases. This can be done by properly choosing control parameters $c_{p}, c_{I}$ and $k_{1}$ as shown in the following.

Let

$$
k_{3}=k_{1} \text {. }
$$

Since the engine/brake dynamics are much faster than the vehicle dynamics (which thus can be neglected in the stage of vehicle performance analysis), the vehicle dynamics of the closed loop system under either throttle control (28), (28) or brake control (29), (30) can be represented by

$$
\begin{aligned}
& \dot{v}_{i}=\operatorname{Rr} \dot{w}_{e} \\
= & \frac{1}{1+\lambda c_{p}}\left[-\beta_{2}\left(\hat{v}_{i-1}-v_{i-1}\right)-\beta_{1}\left(\hat{x}_{i-1}-x_{i-1}\right)\right. \\
& +\left(c_{p}+k_{1}\right)\left(\hat{v}_{i-1}-v_{i}\right)+\left(c_{I}+k_{1} c_{p}\right) \hat{\delta}_{i} \\
& \left.+k_{1} c_{I} \int_{0}^{t} \hat{\delta}_{i} d z\right]
\end{aligned}
$$

From the definition of $\delta_{i}(9)$ and $(30)$, we have

$$
\begin{aligned}
& \left(1+\lambda c_{p}\right) \dddot{\hat{\delta}}_{i} \\
= & \left(1+\lambda c_{p}\right)\left(\ddot{\hat{v}}_{i-1}-\ddot{v}_{i}-\lambda \dddot{v}_{i}\right) \\
= & \left(1+\lambda c_{p}\right) \ddot{\hat{v}}_{i-1}-\left[-\beta_{2}\left(\dot{\hat{v}}_{i-1}-\dot{v}_{i-1}\right)-\beta_{1}\left(\hat{v}_{i-1}-v_{i-1}\right)\right. \\
& \left.+\left(c_{p}+k_{1}\right)\left(\dot{\hat{v}}_{i-1}-\dot{v}_{i}\right)+\left(k_{1} c_{p}+c_{I}\right) \dot{\hat{\delta}}_{i}+k_{1} c_{I} \hat{\delta}_{i}\right] \\
& -\lambda\left[-\beta_{2}\left(\hat{\hat{v}}_{i-1}-\ddot{v}_{i-1}\right)-\beta_{1}\left(\operatorname{dot} v_{i-1}-\dot{v}_{i-1}\right)\right. \\
& \left.+\left(c_{p}+k_{1}\right)\left(\ddot{\hat{v}}_{i-1}-\ddot{v}_{i}\right)+\left(k_{1} c_{p}+c_{I}\right) \ddot{\hat{\delta}}_{i}+k_{1} c_{I} \dot{\hat{\delta}}_{i}\right]
\end{aligned}
$$

Therefor, we have the following relationship:

$$
\begin{aligned}
& s_{i}(0) \\
& =\frac{\left[\left(1+\lambda c_{p}+\lambda \beta_{2}\right) 0^{2}+\left(\beta_{2}+\lambda \beta_{1}\right) \cdot+\beta_{1}\right] \theta_{i-1}(0)}{\left(1+\lambda c_{p}\right) \cdot 0+\left(\lambda c_{I}+\lambda k_{1} c_{p}+c_{p}+k_{1}\right) p^{2}+\left(\lambda k_{1} c_{I}+c_{I}+k_{1} c_{p}\right) \cdot+k_{1} c_{I}}
\end{aligned}
$$

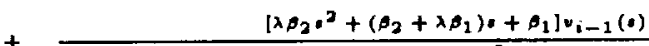

$$
\begin{aligned}
& +\overline{\left(1+\lambda c_{p}\right) 0^{3}+\left(\lambda c_{I}+\lambda k_{1} c_{p}+c_{p}+k_{1}\right) \rho^{3}+\left(\lambda k_{1} c_{I}+c_{I}+k_{1} c_{p}\right)=+k_{1} c_{I}}
\end{aligned}
$$

Furthermore, from (22), we have stable transfer function

$$
\frac{\hat{v}_{i-1}(s)}{v_{i-1}(s)}=\frac{\beta_{2} s+\beta_{1}}{s^{2}+\beta_{2} s+\beta_{1}}
$$

From (32) and (33), we conclude that, by properly choosing design parameters $c_{p}, c_{I}$ and $k_{1}$, we can make $\hat{\delta}_{i}$ converge to zero if $\dot{v}_{i-1}$ is constant, (i.e., if the preceding vehicle is traveling at constant acceleration) and have satisfactory transient response of $\hat{\delta}_{i}$.

From the definitions of $\delta_{i}(9)$ and $\hat{\delta}_{i}(23)$, we see

$$
\delta_{i}=\hat{\delta}_{i}+x_{i-1}-\hat{x}_{i-1}
$$

As pointed out in Remark 4.1, $x_{i-1}-\hat{x}_{i-1}$ will converge to zero exponentially under the condition $\dot{v}_{i-1}=0$. It follows that $\delta_{i}$ will converge to zero while the preceding vehicle is traveling at constant speed.

\section{Asymptotic Platoon Stability}

In this section, we will show that by properly choosing design parameters, the controller proposed in Section 5 can achieve asymptotic platoon stability when it is installed on each vehicle of a group of vehicles (one following another) with safe distance rule (8).

\section{Asymptotic Platoon Stability}

Consider a group of vehicles all equipped with the proposed throttle controller (28) and brake controller (29). Since, at steady state of vehicle following,

$$
\hat{v}_{i-1}=v_{i-1} \text { and } \hat{\delta}_{i}=\delta_{i},
$$

we see from (30)

$$
\begin{aligned}
& \dot{v}_{i} \\
= & \frac{1}{1+c_{p} \lambda}\left[\left(c_{p}+k_{1}\right)\left(v_{i-1}-v_{i}\right)+\left(c_{I}+k_{1} c_{p}\right) \delta_{i}\right. \\
& \left.+k_{1} c_{I} \int_{0}^{t} \delta_{i}(z) d z\right] \\
= & c_{p} \delta_{i}+\left(k_{1} c_{p}+c_{I}\right) \delta_{i}+k_{1} c_{I} \int_{0}^{t} \delta_{i} d t+k_{1}\left(v_{i-1}-v_{i}\right)
\end{aligned}
$$


Differentiating equation (23) three times and substituting the derivative of $v_{i}$ by (34), we obtain

$$
\begin{aligned}
& \dddot{\delta}_{i}(t) \\
= & \ddot{v}_{i-1}-\ddot{v}_{i}-\lambda \dddot{v}_{i} \\
= & c_{p} \ddot{\delta}_{i-1}+\left(k_{1} c_{p}+c_{I}\right) \dot{\delta}_{i-1}(t)+k_{1} c_{I} \delta_{i-1}+k_{1}\left(\dot{v}_{i-2}-\dot{v}_{i-1}\right) \\
& -\left[c_{p} \ddot{\delta}_{i}+\left(k_{1} c_{p}+c_{I}\right) \dot{\delta}_{i}(t)+k_{1} c_{I} \delta_{i}+k_{1}\left(\dot{v}_{i-1}-\dot{v}_{i}\right)\right] \\
& -\lambda\left[c_{p} \dddot{\delta}_{i}+\left(k_{1} c_{p}+c_{I}\right) \ddot{\delta}_{i}+k_{1} c_{I} \dot{\delta}_{i}+k_{1}\left(\ddot{v}_{i-1}-\ddot{v}_{i}\right)\right]
\end{aligned}
$$

From the above equation, we obtain the transfer function $\frac{1}{\lambda^{2}}$ from $\delta_{i}$ to $\delta_{i-1}$

$$
\begin{aligned}
& \frac{\delta_{i}(0)}{\delta_{i-1}(0)}:=\sigma_{1}(0)= \\
& \frac{\left(k_{1}+c_{p}\right) \rho^{2}+\left(k_{1} c_{p}+c_{f}\right) \cdot+k_{1} c_{I}}{\left(1+\lambda c_{p}\right) 0^{3}+\left(\lambda c_{I}+\lambda k_{1} c_{p}+c_{p}+k_{1}\right) \rho^{2}+\left(\lambda k_{1} c_{I}+c_{I}+k_{1} c_{p}\right) \cdot+k_{1} c_{I}}
\end{aligned}
$$

To avoid slinky-type effects, the disturbances caused by the lead vehicle in all frequencies should be attenuated along the following vehicles to insure that they do not become unreasonably large by the end. A sufficient condition for this to happen is for all $i$

$$
\left|\frac{\delta_{i}(j w)}{\delta_{i-1}(j w)}\right|=\left|G_{1}(j w)\right|<1, \quad \text { for all } w>0
$$

With $G_{1}(s)$ given in (35), the inequality in (36) yields

$$
\begin{aligned}
& \left|\frac{\left[k_{1} c_{I}-\left(k_{1}+c_{p}\right) w^{2}\right]^{2}+w^{2}\left(k_{1} c_{p}+c_{I}\right)^{2}}{\left.\left[k_{1} c_{I}-\left(\lambda c_{I}+\lambda k_{1} c_{p}+c_{p}+k_{1}\right) w^{2}\right]^{2}+w^{2}\left[\lambda k_{1} c_{I}+c_{I}+k_{1} c_{p}\right)-\left(1+\lambda c_{p}\right) w^{2}\right]^{2}}\right| \\
& \langle 1 \quad \text { for all } w>0
\end{aligned}
$$

Simplifying the above inequality, we get

$$
\begin{aligned}
& \left(1+\lambda c_{p}\right)^{2} w^{4}+\left[\lambda^{2} c_{I}^{2}+\lambda^{2} k_{1}^{2} c_{p}^{2}\right. \\
& \left.+2 \lambda k_{1} c_{p}^{2}-2\left(c_{I}+k_{1} c_{p}\right)\right] w^{2}+\lambda^{2} k_{1}^{2} c_{I}^{2}>0 \\
& \text { for all } w>0
\end{aligned}
$$

A sufficient condition such that (37) holds is

$$
\lambda^{2} c_{I}^{2}+\lambda^{2} k_{1}^{2} c_{p}^{2}+2 \lambda k_{1} c_{p}^{2}-2\left(c_{I}+k_{1} c_{p}\right)>0
$$

or equivalently

$$
\frac{\left(c_{I}-\frac{1}{\lambda^{2}}\right)^{2}}{A^{2}}+\frac{\left(c_{p}-\frac{k_{1}}{\lambda^{2} k_{1}^{2}+2 \lambda k_{1}}\right)^{2}}{B^{2}}>1
$$

where

$$
\begin{aligned}
A^{2} & =\frac{2 \lambda k_{1}+2}{\lambda^{4}\left(\lambda k_{1}+2\right)} \\
B^{2} & =\frac{2 \lambda k_{1}+2}{\lambda^{3} k_{1}\left(\lambda k_{1}+2\right)}
\end{aligned}
$$

Given $k_{1}>0$ and $\lambda>0$, the suitable values of parameters $c_{I}$ and $c_{p}$ satisfying inequality (38) reside outside shaded ellipse as shown in Figure 2. Consequently, if we choose $c_{I}, k_{1}, c_{p}$ outside the shaded ellipse as shown in Figure 2, asymptotic platoon stability can be assured.

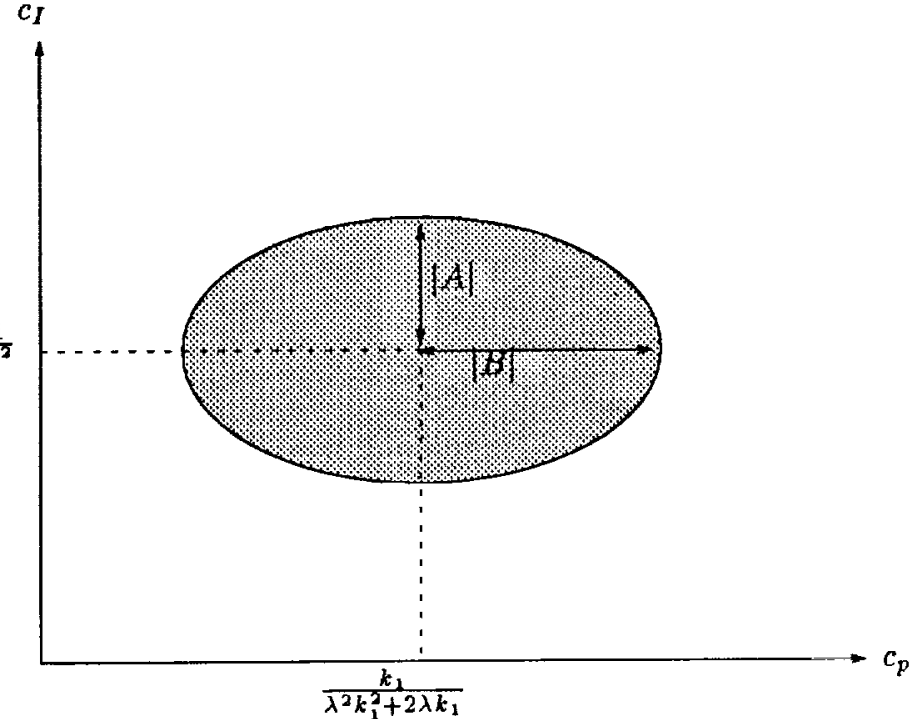

Figure 2: Parameter region for avoiding slinky effects

Remark 6.1 When constant spacing safety policy $(\lambda=$ 0 ) is adopted, inequality (37) for avoiding slinkytype effects reduces to

$$
w^{2}-2\left(c_{I}+k_{1} c_{p}\right)>0
$$

Since $c_{I}+k_{1} c_{p}>0$ (to insure all the poles of $G_{1}(s)$ are in the open left half complex plane), the above inequality can not be satisfied when $w^{2}<$ $2\left(c_{I}+k_{1} c_{p}\right)$. In other words, asymptotic stability can not be assured for low frequency disturbances under constant spacing safety distance policy.

Asymptotic platoon stability under sensor delays In this subsection, the relationships between the sensor delays, the gains of the proposed controller, and the asymptotic platoon stability will be investigated. The results obtained in this subsection can be used to quantify the performance requirements for the sensors for a specific designed controller.

Let $\tau$ be the time delay caused by the velocity sensor and the position sensor, such that the velocity and position terms in (28) and (30) are functions for $t-\tau$ instead of $t$. Then the vehicle dynamics of the closed loop system can be represented by

$$
\begin{aligned}
\dot{v}_{i}(t)= & \frac{1}{1+c_{p} \lambda}\left[\left(c_{p}+k_{1}\right)\left(v_{i-1}-v_{i}\right)(t-\tau)+\left(c_{I}+k_{1} c_{p}\right) \delta_{i}(t-\tau)\right. \\
& \left.+k_{1} c_{I} \int_{0}^{t} \delta_{i}(\xi-\tau) d \xi\right]
\end{aligned}
$$


Differentiating both sides of $(9)$ three times, we get

$$
\begin{aligned}
& \dddot{\delta}_{i}(t) \\
= & \ddot{v}_{i-1}(t)-\ddot{v}_{i}(t)-\lambda \dddot{v}_{i}(t) \\
= & c_{p} \dddot{\delta}_{i-1}(t-\tau)+\left(k_{1} c_{p}+c_{I}\right) \dot{\delta}_{i-1}(t-\tau) \\
& +k_{1} c_{I} \delta_{i-1}(t-\tau)+k_{1}\left(\dot{v}_{i-2}(t-\tau)-\dot{v}_{i-1}(t-\tau)\right) \\
& -\left[c_{p} \ddot{\delta}_{i}(t-\tau)+\left(k_{1} c_{p}+c_{I}\right) \dot{\delta}_{i}(t-\tau)+k_{1} c_{I} \delta_{i}(t-\tau)\right. \\
& \left.+k_{1}\left(\dot{v}_{i-1}(t-\tau)-\dot{v}_{i}(t-\tau)\right)\right]-\lambda\left[c_{p} \dddot{\delta}_{i}(t-\tau)\right. \\
& +\left(k_{1} c_{p}+c_{I}\right) \ddot{\delta}_{i}(t-\tau)+k_{1} c_{I} \dot{\delta}_{i}(t-\tau) \\
& \left.+k_{1}\left(\ddot{v}_{i-1}(t-\tau)-\ddot{v}_{i}(t-\tau)\right)\right]
\end{aligned}
$$

Substituting (39) into (40) and taking Laplace transforms, we can derive the transfer function from $\delta_{i-1}$ to $\delta_{i}$

$$
\begin{aligned}
& \frac{\delta_{i}(s)}{\delta_{i-1}(s)}:=G_{2}(0)= \\
& \frac{\left(k_{1}+c_{p}\right) s^{2}+\left(k_{1} c_{p}+c_{j}\right) \cdot+k_{1} c_{I}}{\left(e^{i r}+\lambda c_{p}\right) s^{3}+\left(\lambda c_{I}+\lambda k_{1} c_{p}+c_{p}+k_{1}\right) 0^{2}+\left(\lambda k_{1} c_{I}+c_{I}+k_{1} c_{p}\right)+k_{1} c_{I}}
\end{aligned}
$$

A sufficient condition for asymptotic stability is, for all $i$

$$
\left|G_{2}(j w)\right|^{2}<1, \quad \text { for all } w>0
$$

Substituting (41) into the above inequality, we obtain

$$
\begin{aligned}
& \left|\frac{\left(d-e w^{2}\right)^{2}+f^{2} w^{2}}{\left[d-b w^{2}+(\sin w \tau) w^{3}\right]^{2}+w^{2}\left[c-\left(a+\cos (w \tau) w^{2}\right]^{2}\right.}\right|<1, \\
& \quad \text { for all } w>0
\end{aligned}
$$

where

$$
\left.\begin{array}{rl}
a & =\lambda c_{p}, \quad b=\lambda c_{I}+\lambda k_{1} c_{p}+c_{p}+k_{1} \\
c & =\lambda k_{1} c_{I}+c_{I}+k_{1} c_{p}, \quad d=k_{1} c_{I}
\end{array}\right)
$$

With equations in (43), condition for asymptotic platoon stability (42) is equivalent to

$$
\begin{aligned}
& {\left[a^{2}+2 a \cos (w \tau)+1\right] w^{4}-2 b \sin (w \tau) w^{3}} \\
& +\left[b^{2}-2 a c-2 c \cos (w \tau)-e^{2}\right] w^{2}+2 d \sin (w \tau) w \\
& +\left(c^{2}-f^{2}-2 b d+2 d e\right)>0, \quad w>0
\end{aligned}
$$

Proposition 5 Consider the vehicle longitudinal system (3) - (6) with control law (28), (29). The asymptotic platoon stability is guaranteed if

$$
\begin{aligned}
& \tau<\min \left\{\frac{\left(\lambda c_{p}-1\right)^{2}}{2\left(\lambda c_{I}+\lambda k_{1} c_{p}+c_{p}+k_{1}\right)},\right. \\
& \left.\frac{\lambda^{2} c_{I}^{2}+\lambda^{2} k_{1}^{2} c_{p}^{2}+2 \lambda k_{1} c_{p}^{2}-2\left(c_{I}+k_{1} c_{p}\right)}{2 k_{1} c_{I}}\right\}
\end{aligned}
$$

Remark 6.2 In Subsection 4.2, we have chosen $\lambda^{2} c_{I}^{2}+$ $\lambda^{2} k_{1}^{2} c_{p}^{2}+2 \lambda k_{1} c_{p}^{2}-2\left(c_{I}+k_{1} c_{p}\right)$ to be positive to insure asymptotic platoon stability. Furthermore, $\lambda c_{I}+\lambda k_{1} c_{p}+c_{p}+k_{1}$ and $k_{1} c_{I}$ are also chosen to be positive to guarantee local stability (regulation of $\delta_{i}$ ). Therefore, the right hand side of inequality (45) is positive.
Proof: Since the inequality in (44) can be rewritten as

$$
\begin{aligned}
& \left\{\left[a^{2}+2 a \cos (w \tau)+1\right] w-2 b \sin (w \tau)\right\} w^{3} \\
& +\left\{\left[b^{2}-2 a c-2 c \cos (w \tau)-e^{2}\right] w+2 d \sin (w \tau)\right\} w \\
& +\left(c^{2}-f^{2}-2 b d+2 d e\right)>0,
\end{aligned}
$$

asymptotic platoon stability is guaranteed if

$$
\begin{aligned}
& {\left[a^{2}+2 a \cos (w \tau)+1\right] w-2 b \sin (w r)>0,} \\
& {\left[b^{2}-2 a c-2 c \cos (w \tau)-e^{2}\right] w+2 d \sin (w \tau)>0, \text { and }(47)} \\
& c^{2}-f^{2}-2 b d+2 d e>0
\end{aligned}
$$

It is easily verified that

$$
\frac{(a-1)^{2}}{2 b}=\frac{\left(\lambda c_{p}-1\right)^{2}}{2\left(\lambda c_{I}+\lambda k_{1} c_{p}+c_{p}+k_{1}\right)}
$$

and

$$
\frac{b^{2}-2 a c-2 c-e^{2}}{2 d}=\frac{\lambda^{2} c_{I}^{2}+\lambda^{2} k_{1}^{2} c_{p}^{2}+2 \lambda k_{1} c_{p}^{2}-2\left(c_{I}+k_{1} c_{p}\right)}{2 k_{1} c_{I}}
$$

such that condition (45) is equivalent to

$$
<\min \left\{\frac{(a-1)^{2}}{2 b}, \frac{b^{2}-2 a c-2 c-e^{2}}{2 d}\right\} .
$$

Since $a>0$ and $b>0$, we see from (49)

$$
\begin{aligned}
a^{2}+2 a \cos (w \tau)+1 \geq a^{2}-2 a+1>2 b \tau \geq 2 b \frac{\sin (\tau w)}{w}, \\
b^{2}-2 a c-2 c \cos (w \tau)-e^{2} \geq b^{2}-2 a c-2 c-e^{2}>2 d \tau \\
\geq-2 d \frac{\sin (\tau w)}{w}
\end{aligned}
$$

which guarantee the inequalities (46) and (47). Moreover, from (43), we see

$$
c^{2}-f^{2}-2 b d+2 d e=\lambda^{2} k_{1}^{2} c_{I}^{2}>0
$$

which assures the inequality (48).

\section{Simulation Results}

We consider vehicles following each other in a single lane with no passing. Each vehicle is assumed to be equipped with the proposed controller. The length of vehicles is assumed to be 4 meters. The following controller gains were selected for the simulations :

$$
\begin{aligned}
& c_{p}=2, \quad c_{I}=0.5, \quad c_{v}=2, \\
& k_{1}=5, \quad k_{2}=40, \quad k_{3}=5, \quad k_{4}=1, \lambda=1, \quad \lambda_{3}=2 .
\end{aligned}
$$

Case 1: Vehicle following with zero initial conditions: Six vehicles are assumed to follow each other and form a platoon in a single lane. The leading vehicle is assumed to accelerate from $9 \mathrm{~m} / \mathrm{sec}$ to $15 \mathrm{~m} / \mathrm{sec}$, then to 21 $\mathrm{m} / \mathrm{sec}$, and then to $27 \mathrm{~m} / \mathrm{sec}$. After achieves $27 \mathrm{~m} / \mathrm{sec}$, it then decelerates to $21.5 \mathrm{~m} / \mathrm{sec}$ and then to $17 \mathrm{~m} / \mathrm{sec}$. Zeroinitial conditions are assumed. The simulation results are shown in Figure (3). Good velocity tracking, small transient spacing error and zero steady state spacing error are achieved for each vehicle. Moreover, no slinky-type effects exist. In other words, asymptotic platoon stability is achieved. 
Case 2 : Exit from the automatic lane: The following situation is considered : at time $t=0 \mathrm{sec}$, the leading vehicle changes lanes and the new vehicle target is $3.2 \mathrm{~m} / \mathrm{s}$ faster and meters farther ahead than the previous one. In this situation, a suddenly change of the relative velocity and relative distance appears which is then confirmed by the on-board computer and the automatic control equipment is reset. Thus, non-zero initial conditions appear. The velocity, acceleration, and spacing deviation profiles shown in Figure (4) are quite smooth during the transient stage.

\section{CONCLUSION}

In this paper, we have studied the vehicle following control problem for the autonomous intelligent vehicles under the constant time headway safety distance rule. Instead of using simplified linear vehicle following models frequently used in vehicle longitudinal control, we consider a nonlinear model that contains important attributes of engines dynamics. Using a newly developed nonlinear control technique, we are able to design throttle and brake controllers for the longitudinal control purpose with smooth maneuvers. One of features of this design is that the asymptotic platoon stability can be achieved with properly chosen design parameters. We further show that this nice property is theoretically robust to a certain degree of sensor delays. The computer simulation results demonstrate the effectiveness of our control approach and enhance the feasibility of practical AICC technology deployment.

Acknowlegement The authors would like to thank Professor Hedrick and Mr. Swaroop for providing the data of the vehicle engine system and several useful comments.

\section{REFERENCES}

[1] H. Y. Chiu, G. B. Stupp, and S. J. Brown. Vehicle-follower controls with variable-gains for short-headway automated guideway transit systems. ASME Journal of Dynamic System, Measurement and Control, 99:183-189, 1977.

[2] R. A. Freeman and P. V. Kokotovic. Backstepping design of robust controllers for a class of nonlinear systems. preprint, 1991.

[3] J. K. Hedrick, D. McMahon, V. Narendran, and D. Swaroop. Longitudinal vehicle controller design for ivhs systems. American Control Conference, pages $3107-3112,1991$.

[4] P. Ioannou and C. C. Chien. Autonomous intelligent cruise control. to appear in IEEE Transactions on Vehicular Technology, 1993.
[5] J. J. Moskwa and J. K. Hedrick. Modeling and validation of automotive engines for control algorithm development. ASME Journal of Dynamic System, Measurement and Control, pages 278-285, 1992.

[6] A. J. Pue. A state-constraint approach to vehiclefollower control for short headway automated transit vehicles. ASME Journal of Dynamic System, Measurement and Control, 99:183-189, 1977.

[7] Shahab Sheikholeslam and Charles A. Desoer. A system level study of the longitudinal control of a platoon of vehicles. ASME Journal of Dynamic System, Measurement and Control, 114:286-292, 1992.

[8] S. Shladover. Operation of automated guideway transit vehicles in dynamically reconfigured trains and platoons. UMTA-MA-06-0085-79, 1979.

[9] D. Swaroop, C. C. Chien, P. Ioannou, and J. K. Hedrick. A comparision of spacing and headway control laws for automatically controlled vehicle. submitted to Journal of Vehicle System Dynamics, 1993.

[10] J. Y. Wong. Theory of ground vehicles. John Wiley $\mathcal{E}$ Sons, NY, 1978. 

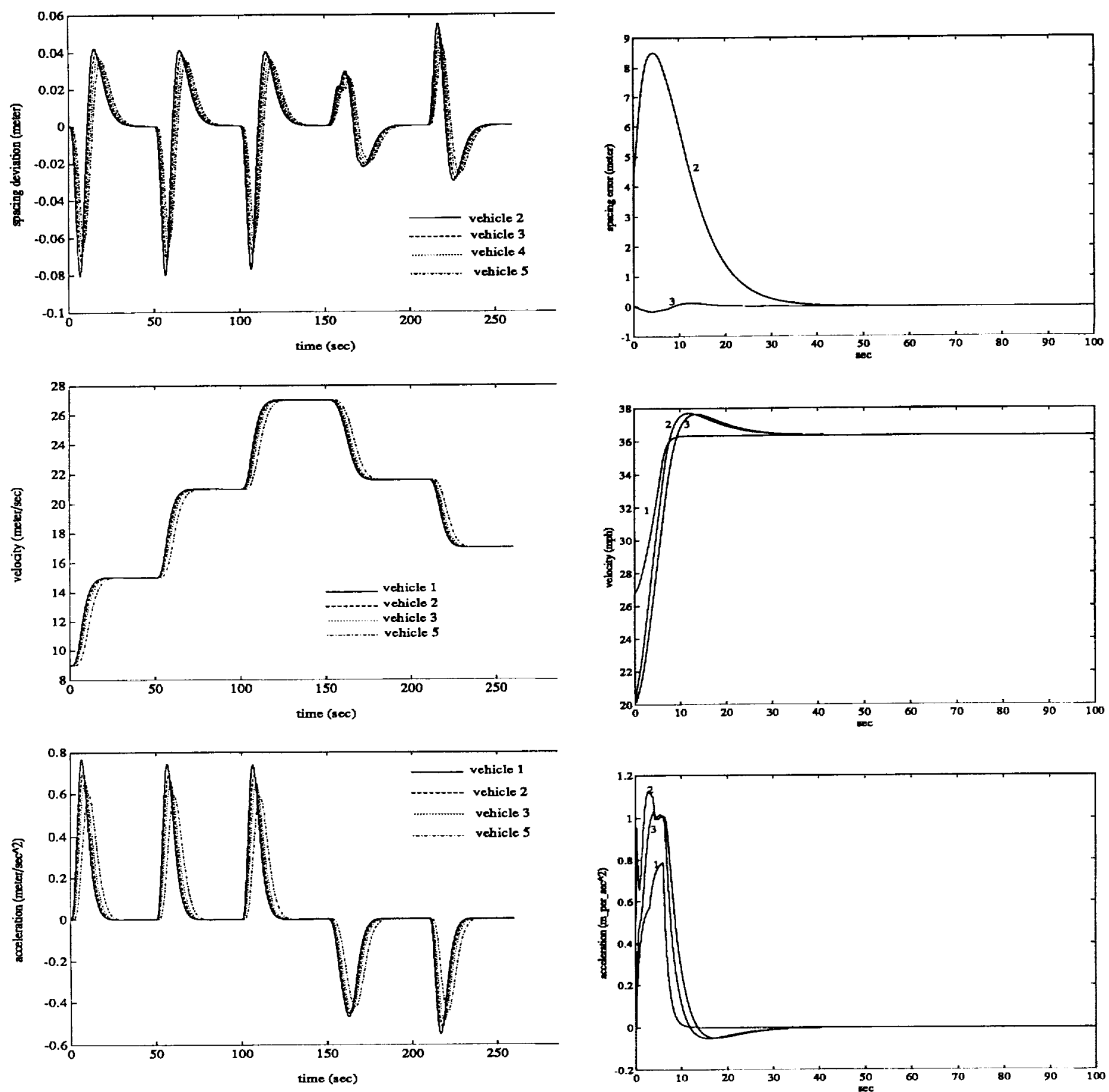

Figure 3: Case 1: Spacing deviation, velocity and acceleration profiles for a vehicle following maneuver with zero initial conditions

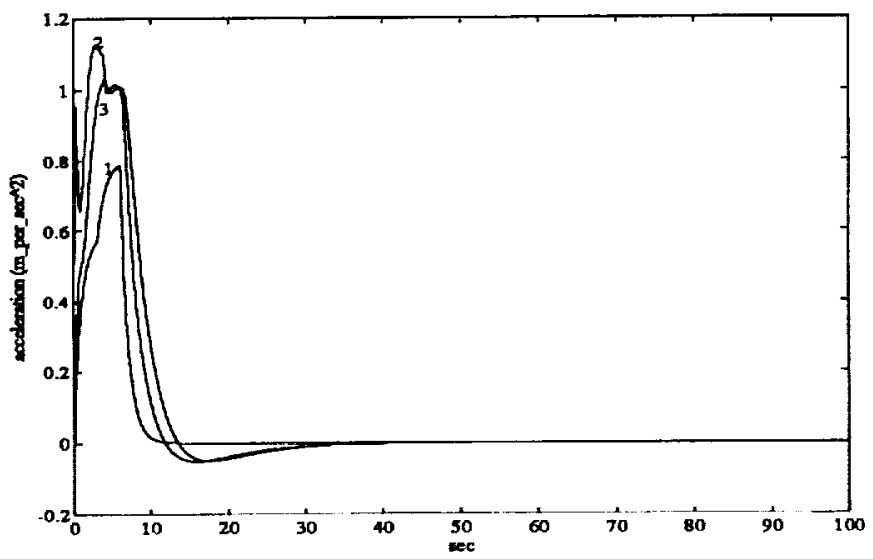

Figure 4: Case 2: Auto-exit situation 